\title{
Multipartite Hypergraphs Achieving Equality in Ryser's Conjecture
}

\author{
Ron Aharoni ${ }^{1}$ • János Barát ${ }^{2,3}$ - Ian M. Wanless ${ }^{2}$
}

Received: 14 October 2014 / Revised: 24 March 2015 / Published online: 30 April 2015 (C) Springer Japan 2015

\begin{abstract}
A famous conjecture of Ryser (1967) is that in an $r$-partite hypergraph the covering number is at most $r-1$ times the matching number. If true, this is known to be sharp for $r$ for which there exists a projective plane of order $r-1$. We show that the conjecture, if true, is also sharp for the smallest previously open value, namely $r=7$. For $r \in\{6,7\}$, we find the minimal number $f(r)$ of edges in an intersecting $r$-partite hypergraph that has covering number at least $r-1$. We find that $f(r)$ is achieved only by linear hypergraphs for $r \leq 5$, but that this is not the case for $r \in\{6,7\}$. We also improve the general lower bound on $f(r)$, showing that $f(r) \geq 3.052 r+O(1)$. We show that a stronger form of Ryser's conjecture that was used to prove the $r=3$ case fails for all $r>3$. We also prove a fractional version of the following stronger form of Ryser's conjecture: in an $r$-partite hypergraph there exists a set $S$ of size at most $r-1$, contained either in one side of the hypergraph or in an edge, whose removal reduces the matching number by 1 .
\end{abstract}

Keywords Intersecting hypergraph · Covering number · Ryser's conjecture · Fractional cover

Research supported by ARC Grant DP120100197, OTKA Grant NN.114614, BSF Grant 2018106 and ISF Grant 1581/12.

$凶 \quad$ Ian M. Wanless

Ian.Wanless@monash.edu

1 Department of Mathematics, Technion, 32000 Haifa, Israel

2 School of Mathematical Sciences, Monash University, Clayton, VIC 3800, Australia

3 Present Address: MTA-ELTE Geometric and Algebraic Combinatorics Research Group, Budapest, Hungary 


\section{Introduction}

For a hypergraph $H$ we use $|H|$ to denote the number of edges (also called lines) and $|V(H)|$ for the number of vertices. A hypergraph is $r$-uniform if every edge has $r$ vertices on it. We use $\mathcal{P}_{r}$ to denote any $r$-uniform projective plane. In the standard terminology of projective planes, $\mathcal{P}_{r}$ has order $r-1$.

A $k$-cover of a hypergraph is a set of $k$ vertices meeting every edge of the hypergraph. The covering number $\tau(H)$ of a hypergraph $H$ is the minimum $k$ for which there is a $k$-cover of $H$. A matching is a set of disjoint edges, and the matching number $v(H)$ of a hypergraph $H$ is the maximum size of a matching consisting of edges of $H$. A hypergraph with $v(H)=1$ is said to be intersecting. An intersecting hypergraph is linear (also called almost disjoint) if each pair of distinct edges meets in exactly one vertex. In an $r$-uniform hypergraph $\tau \leq r v$, since a cover can be obtained from the union of all edges in a matching that is maximal with respect to containment. This bound is sharp, as shown by $\mathcal{P}_{r}$, or by the union of disjoint copies of $\mathcal{P}_{r}$. Sharpness is also attained by many other examples, such as the set of all subsets of size $r$ in a ground set of size $k r-1$, which has $v=k-1$ and $\tau=(k-1) r$.

A hypergraph is $r$-partite if its vertex set $V$ can be partitioned into $r$ sets $V_{1}, \ldots, V_{r}$, called the sides of the hypergraph, so that every edge contains precisely one vertex from each side. In particular, $r$-partite hypergraphs are $r$-uniform. Ryser [17] conjectured the following:

Conjectute 1.1 In an $r$-partite hypergraph, $\tau \leq(r-1) \nu$.

Very little is known about this conjecture. In [2] it was proved for $r=3$. For $r=4,5$ it was shown in [11] that there exists $\epsilon>0$ such that that $\tau<(r-\epsilon) v$ in every $r$-partite hypergraph.

There is only one family of $r$-partite hypergraphs known to attain Ryser's bound: subhypergraphs of truncated projective planes. Denoted by $\mathcal{P}_{r}^{\prime}$, the truncated projective plane of uniformity $r$ is obtained from $\mathcal{P}_{r}$ by the removal of a single vertex $v$ and the edges containing $v$. The sides of $\mathcal{P}_{r}^{\prime}$ are the sets of vertices other than $v$ on the edges of $\mathcal{P}_{r}$ containing $v$. To achieve equality in Ryser's conjecture it is enough to take only a small proportion of the edges of $\mathcal{P}_{r}^{\prime}$. Kahn [12] proved:

Theorem 1.2 A random set of $22 r \log r$ lines in an $r$-uniform projective plane satisfies $\tau=r$ with probability tending to 1 as $r \rightarrow \infty$.

This implies that:

Theorem 1.3 A random set of $22 r \log r$ lines in $\mathcal{P}_{r}^{\prime}$ satisfies $\tau \geq r-1$ with probability tending to 1 as $r \rightarrow \infty$.

Solving an old problem of Erdős and Lovász, Kahn [12] proved that there exist $r$-uniform intersecting hypergraphs with linearly many edges, satisfying $\tau=r$. Mansour et al. [16] conjectured that something similar is true in the $r$-partite case. They defined $f(r)$ to be the smallest integer $k$ for which there exists an $r$-partite intersecting hypergraph $H$ with $k$ edges and $\tau(H) \geq r-1$, and conjectured that $f(r) \leq O(r)$. If the hypergraphs constructed in [12] were part of $\mathcal{P}_{r}$, then this result would imply 
a linear bound on $f(r)$ for infinitely many values, but unfortunately this is not the case. It is not even clear whether $f(r)$ exists for all $r$, since it is conceivable that there is no hypergraph with the required properties. If $r-1$ is a prime power then $\mathcal{P}_{r}^{\prime}$ is known to exist, providing proof that $f(r)$ is defined, but examples for other $r$ were previously unknown. The first case for which the existence of $f(r)$ was previously unknown is $r=7$. In Sect. 2 we prove that $f(7)$ exists and that in fact $f(7)=17$. We also calculate $f(6)$ and improve the general lower bound on $f(r)$. We show that for $r \leq 5$ all hypergraphs attaining $f(r)$ are linear. In contrast, there are non-linear hypergraphs that achieve $f(6)$ and $f(7)$. We finish Sect. 2 by stating a number of open problems.

In Sect. 3 we consider various possible strengthenings of Ryser's conjecture. In particular, a conjecture specifying the form of the desired cover, which in the intersecting case is that the cover of size $r-1$ can be assumed to be contained either in a side or in an edge. We show that a "biased" version of the conjecture, which is true for $r=3$, is false for larger $r$. However, its fractional formulation is true in a strong sense that provides also a fractional version of the above conjecture on the form of the covers. We also prove a fractional version of a strengthening of Ryser's conjecture suggested by Lovász.

\section{How Many Edges are Needed to Achieve $\tau \geq r-1$ ?}

In this section we study the function $f(r)$, defined in the introduction. In particular, we establish the values of $f(6)$ and $f(7)$ and improve the lower bound on $f(r)$ proved in [16]. It is likely that Ryser's conjecture (if true) is sharp for all values of $r$, but so far this has been shown only for $r$ for which $r-1$ is a prime power. The example below shows sharpness for the first open case, $r=7$.

$\begin{array}{llllll}1111111 & 1235354 & 2313664 & 4412343 & 6142564 \\ 2154322 & 1344433 & 3514555 & 4551234 & \\ 3332221 & 1424266 & 3655163 & 5123253 & \\ 4325512 & 2222135 & 4136465 & 5361365 & \end{array}$

Here each sequence describes one edge, where the $i$-th symbol in the sequence indicates which vertex is taken in the $i$-th side $V_{i}$. The above example has 17 edges and 42 vertices, 6 on each side. We used a computer to check that it has no 5-cover, from which it follows that $\tau=6=r-1$.

Remark 2.1 Independently, Abu-Khazneh and Pokrovskiy [1] showed that $f(7)$ exists. The bound they obtained was $f(7) \leq 22$.

Our next aim is to study $f(r)$ for some small values of $r$. A common concept will be the idea of a greedy cover, which is a cover obtained iteratively by including a vertex of maximum degree in the hypergraph induced by the lines that have not yet been covered. Note that in an intersecting hypergraph $H$ with more than one line there is always a vertex of degree at least 2 . Hence there is always a greedy cover of size at most $\lceil|H| / 2\rceil$. If we have information about the degrees of vertices in $H$ we 
can usually find a smaller greedy cover. The next few lemmas will also recur in our calculations.

Lemma 2.2 Let $H$ be an intersecting $r$-partite hypergraph with covering number $\tau$. Suppose $H$ has maximum degree no more than 4 and for $i=1,2,3,4$ let $x_{i}$ denote the number of vertices of degree $i$ in $H$. Then

$$
\begin{aligned}
x_{1}+x_{4} & \geq\left(\begin{array}{c}
|H| \\
2
\end{array}\right)+3 r \tau-2 r|H|, \\
x_{3}+3 x_{4} & \geq\left(\begin{array}{c}
|H| \\
2
\end{array}\right)+r \tau-r|H| .
\end{aligned}
$$

In each of (2) and (3) equality holds if and only if $H$ is linear and has exactly $\tau$ vertices on each side.

Proof Since each side is a cover there are at least $\tau$ vertices in every side. Therefore

$$
x_{1}+x_{2}+x_{3}+x_{4} \geq r \tau .
$$

Counting the pairs $(v, e)$ such that vertex $v$ lies on line $e$ yields,

$$
x_{1}+2 x_{2}+3 x_{3}+4 x_{4}=r|H| \text {. }
$$

Also, every two edges meet, which requires that

$$
x_{2}+3 x_{3}+6 x_{4} \geq\left(\begin{array}{c}
|H| \\
2
\end{array}\right) \text {. }
$$

Now summing (4) and (6) and subtracting (5) we get (3). Similarly, three times (4) plus (6) minus twice (5) gives (2). In both cases, equality requires equality in (4) and (6). The former means that each side has exactly $\tau$ vertices and the latter means that $H$ is linear.

Lemma 2.3 Let $V_{1}$ be one side of an r-partite intersecting hypergraph H. Suppose that $V_{1}$ contains $y_{1}$ vertices of degree 1 and $y_{2}$ vertices of degree at least 2 . Then $\left(y_{1}+1\right) / 2+y_{2} \geq \tau(H)$.

Proof The lines through vertices of degree 1 in $V_{1}$ can be greedily covered using $\left\lfloor\left(y_{1}+1\right) / 2\right\rfloor$ vertices. The remaining lines of $H$ can be covered by the vertices of degree at least 2 in $V_{1}$.

Lemma 2.4 Let $r$ be odd and suppose that $H$ is an intersecting $r$-partite hypergraph satisfying $|H| \leq r$ and $\tau(H) \geq(r+1) / 2$. Then

- $|H|=r$

- $\tau(H)=(r+1) / 2$.

- Each side of $H$ consists of one vertex of degree 1 and $(r-1) / 2$ vertices of degree 2. 
- Each line of $H$ contains one vertex of degree 1 and $r-1$ vertices of degree 2.

- $H$ is linear.

Proof If $|H| \leq r-1$ or if $H$ has a vertex of degree greater than 2 then $H$ has a greedy cover using at most $(r-1) / 2$ vertices. Hence $|H|=r$ and the maximum degree in $H$ is 2 . Even so, there is a greedy $(r+1) / 2$-cover, so $\tau=(r+1) / 2$. Given that $r$ is odd, each side has at least one vertex of degree 1 , so $x_{1} \geq r$. If any line contains two vertices of degree 1 then it cannot meet the other $r-1$ lines without breaching the maximum degree, hence $x_{1}=r$. The claims about degree sequences of sides and of lines follow. Also, counting intersections we have $r(r-1) / 2$ pairs of lines and $r(r-1) / 2$ degree 2 vertices, so $H$ is linear.

Although we will not need it, it is possible to be even more precise about the structure of $H$ in Lemma 2.4. From what we have shown so far, it is clear that an extra line could be added through all of the degree 1 vertices. We would then have a 2-regular linear intersecting $r$-partite hypergraph $H^{\prime}$ with $r+1$ lines. Such a hypergraph corresponds to a 1-factorisation of the complete graph $K_{r+1}$. Each line in $H^{\prime}$ represents a vertex of $K_{r+1}$ and each side of $H^{\prime}$ represents a 1-factor, with each vertex of $H^{\prime}$ specifying a different pair of vertices of $K_{r+1}$. Moreover, if we take any 1-factorisation of $K_{r+1}$, it will build an $H^{\prime}$ as just described, from which we can remove any one line to get a hypergraph $H$ satisfying the conditions in Lemma 2.4 .

In [16] it was shown that $f(r) \geq\left(3-\frac{1}{\sqrt{18}}\right) r(1-o(1)) \approx 2.764 r(1-o(1))$. We next improve this asymptotic lower bound.

Theorem 2.5 Suppose $H$ is an intersecting $r$-partite hypergraph with covering number $\tau$ and maximum degree $\Delta$. Then

$$
\Delta \geq \begin{cases}2 & \text { if }|H| \geq 2 \\ 3 & \text { if } \tau>(r+1) / 2 \\ 4 & \text { if } \tau>2 r / 3+1 \\ 5 & \text { if } \tau \geq(25 r+23) / 32\end{cases}
$$

Proof Treating the right hand side of (3) as a quadratic in $|H|$ we see that it is minimised when $|H|$ is $r$ or $r+1$. Hence $x_{3}+3 x_{4} \geq r(2 \tau-r-1) / 2$, so $\Delta \geq 3$ whenever $\tau>(r+1) / 2$. (This bound is best possible, as demonstrated by Lemma 2.4).

Suppose that $|V(H)|=r \tau+s$ for some $s \geq 0$. Applying Lemma 2.3 to each side of $H$ we discover that $r \tau \leq\left(x_{1}+r\right) / 2+\left(|V|-x_{1}\right)$ which means that $x_{1} \leq$ $r+2(|V|-r \tau)=r+2 s$.

Assume that $\Delta<4$. Incorporating the $s$ error term into the derivation of (2) we find that

$$
x_{1} \geq\left(\begin{array}{c}
|H| \\
2
\end{array}\right)+3 r \tau+3 s-2 r|H| \geq r(3 \tau-2 r-2)+3 s \text {, }
$$

by again minimising the quadratic in $|H|$. Therefore $r \geq x_{1}-2 s \geq r(3 \tau-2 r-2)$, which implies that $\tau \leq 2 r / 3+1$. 
Next assume that $\Delta<5$. As in the previous case, we strengthen (2) to give

$$
x_{4} \geq\left(\begin{array}{c}
|H| \\
2
\end{array}\right)+3 r \tau+3 s-2 r|H|-x_{1} \geq\left(\begin{array}{c}
|H| \\
2
\end{array}\right)+3 r \tau-2 r|H|-r .
$$

There is some side of $H$ with at least $\mu=\left\lceil x_{4} / r\right\rceil$ vertices of degree 4 on it. Using these vertices in a greedy cover we find that

$$
\tau \leq \mu+\lceil(|H|-4 \mu) / 2\rceil=\lceil|H| / 2\rceil-\mu \leq\lceil|H| / 2\rceil-\frac{1}{r}\left(\begin{array}{c}
|H| \\
2
\end{array}\right)-3 \tau+2|H|+1 .
$$

Maximising the quadratic in $|H|$ for each of the two possible parities of $|H|$, we find that $\tau \leq 25 r / 32+11 / 16+1 /(32 r)<(25 r+23) / 32$.

Hence, using a greedy algorithm that chooses a vertex of highest degree at each step, we can locate an $(r-2)$-cover for any intersecting hypergraph with at most $\left(2 \times \frac{1}{2}+3 \times \frac{1}{6}+4 \times \frac{11}{96}+5 \times \frac{7}{32}\right) r+O(1)$ edges.

Corollary 2.6 $f(r) \geq 293 r / 96+O(1) \geq 3.052 r+O(1)$ as $r \rightarrow \infty$.

Of course, Theorem 2.5 can also be used to find lower bounds on $f(r)$ for specific values of $r$. For example, $f(8) \geq 1+2+2+2+3+3+5=18, f(9) \geq$ $1+2+2+2+2+3+3+5=20$ and $f(10) \geq 1+2+2+2+2+3+3+4+5=24$. In [16] small values of $f(r)$ were studied, including a proof that $12 \leq f(6) \leq 15$. We now determine the value of $f(6)$.

Theorem $2.7 f(6)=13$

Remark 2.8 This was proved independently in [1].

Proof The following is a 6-partite intersecting hypergraph with 30 vertices, 13 edges and $\tau=5$.

$$
\begin{array}{lllll}
111111 & 444114 & 125334 & 241535 & 545421 \\
222211 & 553315 & 213444 & 351224 & \\
333131 & 143252 & 255153 & 514233 &
\end{array}
$$

Since $f(6) \geq 12$, it suffices to now show that any 6-partite intersecting hypergraph $H$ with 12 edges can be covered with 4 vertices. Assume to the contrary that $\tau(H) \geq 5$.

First suppose that $H$ has a vertex $v$ of degree 5 or more. Let $H^{\prime}$ be the hypergraph made from $H$ by removing the lines through $v$. By assumption $\tau\left(H^{\prime}\right) \geq 4$, so Theorem 2.5 shows that $H^{\prime}$ has a vertex $u$ of degree at least 3. Together $u$ and $v$ cover at least 8 of the lines of $H$. The remaining lines can be covered in pairs, using at most two further vertices, contradicting $\tau(H) \geq 5$.

From now on, let $x_{i}$ be the number of vertices in $H$ of degree $i$. If there were two vertices of degree 4 in the same side of $H$ then we can find a 4-cover, as before. Therefore, $x_{4} \leq 6$. If there were four vertices of degree 3 in the same side, then they would form a cover, again a contradiction. Also, if there is a vertex of degree 4 in a side, there can be at most one vertex of degree 3 in the same side. Therefore, $x_{3} \leq 18-2 x_{4}$. 
It follows that $x_{3}+3 x_{4} \leq 24$. By Lemma 2.2, we find that $x_{3}+3 x_{4}=24$ and $H$ is linear. Moreover, $x_{4}=6$. Now, no two vertices between them cover 8 lines, since otherwise the remaining lines could be covered greedily in a 4-cover. It follows that each pair of vertices of degree 4 lie on a common line. There are $\left(\begin{array}{l}6 \\ 2\end{array}\right)=15$ such pairs and only 12 lines, so there are three vertices of degree 4 lying on a common line. These three vertices will cover 10 lines between them since $H$ is linear. The remaining two lines can be covered by a single vertex, so we are done.

In [16] it was shown that $f(7) \geq 14$. We next establish the exact value of $f(7)$.

Theorem $2.9 f(7)=17$.

Proof Suppose that $H$ is a 7-partite intersecting hypergraph with $\tau(H) \geq 6$. In (1) we gave an example showing that $f(7) \leq 17$, so it suffices to show that $|H| \geq 17$. By [16] we know that $|H| \geq 14$. Aiming for a contradiction, we assume that $|H| \leq 16$.

Let $v$ be a vertex of maximum degree in $H$. Removing the edges through $v$ and all resulting isolated vertices we obtain a hypergraph $H^{\prime}$. Let $u$ be a vertex of maximum degree in $H^{\prime}$. From $H^{\prime}$, remove the edges through $u$ and all resulting isolated vertices to obtain a hypergraph $H^{\prime \prime}$. By construction $\tau\left(H^{\prime}\right) \geq 5$, and $\tau\left(H^{\prime \prime}\right) \geq 4$. Also $v$ has degree $|H|-\left|H^{\prime}\right|$ in $H$ and $u$ has degree $\left|H^{\prime}\right|-\left|H^{\prime \prime}\right| \leq|H|-\left|H^{\prime}\right|$ in $H^{\prime}$. By Lemma $2.4,\left|H^{\prime \prime}\right| \geq 7$ so $u$ has degree at most 4 in $H^{\prime}$. By Theorem $2.5, u$ has degree at least 3 in $H^{\prime}$, so $\left|H^{\prime}\right| \geq 10$.

Case 1: $\left|H^{\prime \prime}\right|=7$ and $|H| \leq 15$

The structure of $H^{\prime \prime}$ is prescribed by Lemma 2.4. In particular, $H^{\prime \prime}$ has 4 vertices per side. Let $D$ be the sum of the degrees of the vertices in $V(H) \backslash\left(V\left(H^{\prime \prime}\right) \cup\{u, v\}\right)$. By Lemma 2.3, each side that does not contain $u$ or $v$ contributes at least 3 to $D$. There are 6 such sides if $u$ and $v$ are on the same side. Otherwise $u$ and $v$ are on different sides and those sides each contribute at least 1 to $D$. Therefore $D \geq \min \{2 \times 1+5 \times 3,6 \times 3\}=$ 17. Since $H$ is intersecting, each line from $H \backslash H^{\prime \prime}$ includes a cover of $H^{\prime \prime}$ as well as either $u$ or $v$, and thus contributes at most 2 to $D$. Hence $|H|-\left|H^{\prime \prime}\right| \geq 9$, which is impossible.

Case 2: $\left|H^{\prime \prime}\right| \geq 8$ and $|H| \leq 15$

By Theorem 2.5 we may assume that $u$ has degree 3 in $H^{\prime}, v$ has degree 4 in $H$ and $|H|=15$. Applying Lemma 2.2 we find that $x_{3}+3 x_{4} \geq 42$ in $H$. If there were two vertices of degree 4 on the same side we could use them as $v$ and $u$ and we would end up in Case 1 above. So we may assume that $x_{4} \leq 7$. Thus either $x_{3}=21$ and $x_{4}=7$, or else $x_{3}+x_{4}>28$. Either way, some side has 4 vertices covering at least 13 lines or 5 vertices covering at least 15 lines. Both options lead to a 5-cover.

Case 3: $|H|=16$ and $\left|H^{\prime}\right| \leq 10$ 3.

By the preliminary comments we know $\left|H^{\prime}\right|=10, v$ has degree 6 and $u$ has degree

Suppose $H^{\prime}$ has $x_{i}$ vertices of degree $i$ for $1 \leq i \leq 3$. By Lemma $2.2, x_{1} \geq 10$ with equality only if every side has precisely 5 vertices. If we have equality then there exists a side that contains at least two vertices of degree 1, contradicting Lemma 2.3. So we may assume $x_{1} \geq 11$. By the pigeonhole principle there is a line $e_{1}$ with at 
least two vertices of degree 1 on it. Choose a line $e_{2} \neq e_{1}$, and suppose it meets $e_{1}$ at a vertex $v_{2}$. There are at least $10-3=7$ lines that do not pass through $v_{2}$ and hence meet $e_{2}$ at some other vertex. There are 6 vertices in $e_{2} \backslash\left\{v_{2}\right\}$, so one of them, say $v_{3}$, has degree 3 and does not lie on $e_{1}$. Now consider the at least 5 lines which do not pass through $v_{2}$ or $v_{3}$. They have to meet $e_{1}$ in a vertex of degree greater than 1 other than $v_{2}$. There are at most 4 such vertices, so one of them, say $v_{4}$, covers two of the lines as well as $e_{1}$. In other words, $v_{3}$ and $v_{4}$ together cover 6 lines, and the remaining lines can be covered greedily with only two more vertices, a contradiction.

Case 4: $|H|=16$ and $\left|H^{\prime}\right| \geq 12$

Consider any edge $e \in H$. The other 15 lines of $H$ must meet $e$, so there is a vertex on $e$ of degree at least 4 . Hence the degree of $v$, namely $|H|-\left|H^{\prime}\right|$, must be 4 . No side of $H$ can have a degree sequence containing [4, 4, 4], [4, 4, 3, 3] or [4, 3, 3, 3, 3]. So $x_{4} \leq 14$ and $x_{3} \leq 35-2 x_{4}$. Hence $x_{3}+3 x_{4} \leq 49$, contradicting Lemma 2.2.

Case 5: $|H|=16$ and $\left|H^{\prime}\right|=11$

Here $v$ has degree 5. Each line through $v$ in $H$ includes a cover of $H^{\prime}$ and hence contains at most one vertex that is not in $V\left(H^{\prime}\right) \cup\{v\}$. Hence $|V(H)| \leq\left|V\left(H^{\prime}\right)\right|+6$. By (4) we have $|V(H)| \geq 42$ so $\left|V\left(H^{\prime}\right)\right| \geq 36$.

Starting with the vertices of $H$ and the lines of $H^{\prime}$, consider adding the lines through $v$ one at a time in an arbitrary order. For a given line through $v$, suppose that it includes $a_{i}$ vertices (other than $v$ itself) that are of degree $i$ just before the line is added. Given that $\tau\left(H^{\prime}\right) \geq 5$ and that the lines through any two vertices of degree 1 can be covered by a single vertex, we see that $a_{1} \leq 3$ and that if $a_{0}=1$ then $a_{1} \leq 1$. In other words, $a_{1}+2 a_{0} \leq 3$. Similar reasoning can be applied to lines through $u$ that include $b_{i}$ vertices (other than $u$ ) of degree $i$ before they are added to $H^{\prime \prime}$, showing that $b_{1}+2 b_{0} \leq 5$. These facts will be used repeatedly in the subcases below.

Case 5a: $\left|V\left(H^{\prime}\right)\right|=36$ and $H^{\prime}$ has at least 8 vertices of degree 1

It follows that $|V(H)|=42$, and this can only be achieved by each line through $v$ having $a_{0}=1$ (and thus $a_{1} \leq 1$ ). It follows that $H$ has at least as many vertices of degree 1 as $H^{\prime}$. However, this means that some side of $H$ contains two vertices of degree 1, contradicting Lemma 2.3.

Case 5b: $u$ has degree at most 3

Applying Lemma 2.2 to $H^{\prime}$, we see there is inequality in (4) and hence $x_{3}>13$. However, there cannot be 3 vertices of degree 3 on one side (if there were, they could be used in a greedy 4-cover) so $x_{3}=14$, which in turn means that $\left|V\left(H^{\prime}\right)\right|=36$, and $x_{1}=9$. Hence this case reduces to Case 5 a.

Case 5c: $u$ has degree 4 and $\left|V\left(H^{\prime}\right)\right|=36$

When $u$ has degree 4 , we have $\left|H^{\prime \prime}\right|=7$ so the structure of $H^{\prime \prime}$ is prescribed by Lemma 2.4. In particular, $\left|V\left(H^{\prime \prime}\right)\right|=28$ and $H^{\prime \prime}$ has one vertex of degree 1 on each side.

The only way that $H^{\prime}$ can have 36 vertices is if 3 of the lines through $u$ have $b_{0}=2$, $b_{1} \leq 1$ and the remaining line through $u$ has $b_{0}=1, b_{1} \leq 3$. But this means that $H^{\prime}$ has at least $7+7-6=8$ vertices of degree 1 , so we are in Case 5a.

Case 5d: $u$ has degree 4 and $\left|V\left(H^{\prime}\right)\right| \geq 37$ 
Again $H^{\prime \prime}$ is prescribed by Lemma 2.4. However, this time each line through $u$ has $b_{0}=2, b_{1} \leq 1$. Consequently $\left|V\left(H^{\prime}\right)\right|=37$ and $H^{\prime}$ has at least $7+8-4=11$ vertices of degree 1. Also at least 4 of the lines through $v$ have $a_{0}=1, a_{1} \leq 1$ and the remaining line through $v$ has $a_{1} \leq 3$. But this means that $H$ has at least $11+4-7=8$ vertices of degree 1 . This is a contradiction unless $|V(H)|>42$, but that can only happen if $|V(H)|=43$ and all lines through $v$ have $a_{0}=1$. In this case, there are at least $11+5-5=11$ vertices of degree 1 , so at least one side contradicts Lemma 2.3.

We say that a hypergraph achieves $f(r)$ if it is $r$-partite, has $\tau \geq r-1$ and contains only $f(r)$ edges. It is notable that the examples of hypergraphs achieving $f(r)$ that we gave in (1) and (7) are not linear. We now contrast this with the situation for smaller $r$.

Theorem 2.10 For $r \leq 5$ the only hypergraphs achieving $f(r)$ are linear.

Proof The statement is elementary to check for $r \leq 3$ so we will assume that $r \in\{4,5\}$.

Suppose $H$ is a hypergraph achieving $f(r)$. Suppose $H$ has $x_{i}$ vertices of degree $i$ for each $i$. We assume that $H$ is not linear, so that we get inequality when we apply Lemma 2.2 to $H$.

\section{Case 1: $r=4$}

In this case $|H|=f(4)=6$. There are no vertices of degree 4 or more, otherwise we would have a greedy 2-cover. By Lemma 2.2, $x_{3}>3$. However, there cannot be two vertices of degree 3 on one side, since they would form a 2 -cover. So $x_{3}=4$ and each side has a vertex of degree 3 . Thus each side must have degree sequence $[3,2,1]$ or $[3,1,1,1]$. If any side had the latter option, there would be equality in (6). As we are assuming $H$ is not linear, it follows that every side has degree sequence $[3,2,1]$. Even so, there can only be a single pair of lines that meets twice and all other pairs must meet once. Hence we can find a vertex $v_{3}$ of degree 3 such that the lines through $v_{3}$ are disjoint apart from their intersection at $v_{3}$. Let $v_{2}$ and $v_{1}$ respectively be the vertices of degree 2 and 1 on the same side as $v_{3}$. The union of the lines through $v_{3}$ contains every vertex from $V(H) \backslash\left\{v_{1}, v_{2}\right\}$.

No line of $H$ contains two vertices of degree 1 , since the total of the degrees on a line must be at least $|H|+r-1=9$. Yet each side of $H$ has a vertex of degree 1 , so there must be exactly one vertex of degree 1 on each line through $v_{3}$. Hence, up to isomorphism, the lines through $v_{3}$ are as pictured on the left in Fig. 1, where vertices of degree 1 are shown as solid circles and vertices of higher degree are hollow. By inspection, there is only one way to add the lines through $v_{2}$, yielding the diagram on the right in Fig. 1. However, now the line through $v_{1}$ cannot meet all the other lines, giving a contradiction.
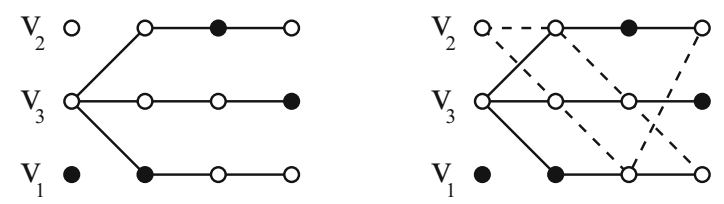

Fig. 1 Diagram for Case 1 of Theorem 2.10 
Case 2: $r=5$

In this case $|H|=f(5)=9$. There are no vertices of degree 5 or more, otherwise we would have a greedy 3 -cover. By Lemma $2.2, x_{3}+3 x_{4}>11$. However, no side can have a degree sequence containing [4, 3] or [3, 3, 3], which means $x_{4} \geq 2$.

Let $v$ be a vertex of degree 4 in $H$. Removing the edges through $v$ and all resulting isolated vertices we obtain a hypergraph $H^{\prime}$ satisfying $\tau\left(H^{\prime}\right) \geq 3$. The structure of $H^{\prime}$ is dictated by Lemma 2.4. Let $L$ denote the lines through $v$ in $H$. Since $H$ is intersecting, each line of $L$ includes a cover of $H^{\prime}$, which means it has at most one vertex outside of $V\left(H^{\prime}\right) \cup\{v\}$. At the same time each side of $H$ must have at least 4 vertices, since $\tau(H) \geq 4$. Therefore each line of $L$ contains a different vertex outside of $V\left(H^{\prime}\right) \cup\{v\}$, which necessarily has degree 1 in $H$. Now the only way to satisfy Lemma 2.3 is if each line in $L$ contains a 3 -cover of $H^{\prime}$ that includes a vertex of degree 1 in $H^{\prime}$. Again, different lines in $L$ must contain different such degree 1 vertices. Therefore, if two lines in $L$ meet at a vertex other than $v$, that vertex has degree 2 in $H^{\prime}$.

Let $u$ be a vertex of degree 4 in $H$, other than $v$. By the above, $u$ has degree 2 in $H^{\prime}$. Removing the edges through $u$ from $H$ and all resulting isolated vertices, we obtain a hypergraph $H^{\prime \prime}$ which must also have the structure in Lemma 2.4. Suppose the lines through $u$ in $H$ are $\ell_{1}, \ell_{2}, \ell_{3}, \ell_{4}$, where $\ell_{1}, \ell_{2} \in H^{\prime}$ and $\ell_{3}, \ell_{4} \in L$. Note that $\ell_{3}$ cannot meet $\ell_{1}$ or $\ell_{2}$ anywhere other than at $u$, since $\ell_{3}$ only has 5 intersections with lines of $H^{\prime}$, counting multiplicities, and has to meet all 5 lines of $H^{\prime}$. A similar statement holds for $\ell_{4}$. Also $\ell_{1}$ and $\ell_{2}$ meet only at $u$ since $H^{\prime}$ is linear. Finally, $\ell_{3}$ and $\ell_{4}$ do not meet at any vertex with degree 2 in $H$. Putting these observations together, we find that all vertices in $V(H) \backslash\left(V\left(H^{\prime \prime}\right) \cup\{u\}\right)$ have degree 1 in $H$. However, $\ell_{1}, \ell_{2}, \ell_{3}, \ell_{4}$ between them contain at most 3 vertices of degree 1 in $H$. This gives the contradiction $20=|V(H)| \leq\left|V\left(H^{\prime \prime}\right)\right|+4=19$.

Many questions remain open about $f(r)$. Mansour et al. [16] conjectured that it grows linearly. Since we have a linear lower bound this is equivalent to:

Conjectute 2.11 $f(r)=O(r)$.

However, that cannot be proved until a much more fundamental question is answered.

\section{Open Problem 1 For which $r$ is $f(r)$ defined?}

We have shown here that $f(7)$ is defined, but the issue is unresolved for all $r>7$ for which $r-1$ is not a prime power. One direction to approach Conjecture 2.11 is to try to find infinitely many $r$ for which $f(r)$ is small. A natural way to try to do this is to find small subsets of $\mathcal{P}_{r}^{\prime}$ with $\tau=r-1$. It is fairly easy to see that approximately half of the lines of a truncated projective plane can be deleted to get a sparser hypergraph with the same $\tau$. Recall that an arc of a projective plane is a set of vertices without three on a line. Conics show that there exist $(q+1)$-arcs in $P G(2, q)$, called ovals. Let $P_{1}, \ldots, P_{r}$ be the vertices of the oval, where $r=q+1$. We delete $P_{r}$ and the lines through it, to get an $r$-partite hypergraph, the truncated projective plane. The sides are identified with the deleted lines $P_{r} P_{i}$, where $1 \leq i \leq r-1$. We delete the lines 
external to the oval. That is, we keep the lines through $P_{1}, \ldots, P_{r-1}$. We kept $r-2$ secants and 1 tangent through each of these $r-1$ vertices. Therefore the number of remaining lines is $\left(q^{2}+q\right) / 2=\left(r^{2}-r\right) / 2$. We claim that $\tau \geq r-1$. Indeed, the degree of any of $P_{1}, \ldots, P_{r-1}$ is $r-1$, therefore the lines through $P_{1}$ cannot be blocked by $P_{2}, \ldots, P_{r-1}$. That is, either the $r-1$ lines through $P_{1}$ are blocked by different vertices or each of $P_{1}, \ldots, P_{r-1}$ is present in the cover. In any case $\tau$ is at least $r-1$. This construction gives an easy way to show that $f(4) \leq 6$ and $f(6) \leq 15$ as shown in [16]. However, Theorem 1.3 shows that it is far from optimal asymptotically. So the challenge remains to find deterministic geometric constructions that do much better, or indeed to show that the random construction is essentially best possible.

Open Problem 2 How small can a subset of the lines of $\mathcal{P}_{r}^{\prime}$ be and still have $\tau=r-1$ ?

Another variant is to insist that a hypergraph be linear, but not necessarily a subset of a projective plane. It is not clear whether being linear helps to achieve $f(r)$ or not.

Open Problem 3 Is $f(r)$ typically achieved by linear hypergraphs, non-linear hypergraphs or both?

In Theorem 2.10 we saw that only linear hypergraphs achieve $f(r)$ for $r \leq 5$. The examples that we gave in (1) and (7) are not linear. However, the following is a 6-partite linear hypergraph with $\tau=5$ :

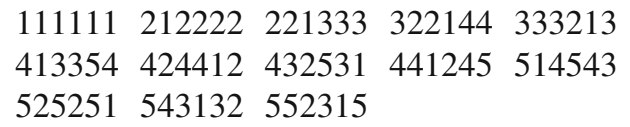

Clearly, $f(6)$ is achieved by both linear and non-linear hypergraphs. We were not able to find a linear hypergraph achieving $f(7)$, and suspect that no such hypergraph exists. Indeed, we were unable to answer the following question:

Open Problem 4 Is there any linear intersecting 7-partite hypergraph with $\tau=6$ ?

Of course, the same question is interesting for other $r$ where $r-1$ is not a prime power. The analogous problem for $r$-uniform hypergraphs is:

Open Problem 5 Is there any linear intersecting 7-uniform hypergraph with $\tau=7$ ?

Next we ask the extent to which Theorem 2.5 generalises.

Open Problem 6 For each positive integer d does there exist an $\epsilon>0$ such that, for sufficiently large $r$, every $r$-partite intersecting hypergraph with $\tau \geq(1-\epsilon) r$ has $\Delta \geq d ?$

Answering this might be one way to improve the lower bound on $f(r)$ given by Corollary 2.6.

In studying $f(r)$ we have concentrated on the case $v=1$, but the same questions can be asked for general $v$. Let $f(r, k)$ be the smallest number of edges in an $r$-partite hypergraph with $v=k$ and $\tau \geq(r-1) k$. Note that $f(r, 1)=f(r)$. Also, we can 
remove edges from any hypergraph satisfying $\tau \geq(r-1) k$ to reach a hypergraph with $\tau=(r-1) k$. It follows that any hypergraph achieving $f(r, k)$ will have $\tau=(r-1) k$. Taking $k$ disjoint copies of an $r$-partite hypergraph with $v=1$ and $\tau=r-1$ shows that $f(r, k) \leq k f(r, 1)$. The results of $[9,10]$ imply that $f(3, k)=k f(3,1)$, for all $k$. Does equality hold more generally?

Open Problem 7 For which $r$ and $k$ is it true that $f(r, k)=k f(r, 1)$ ?

It would also be worth finding bounds or estimates for $f(r, k)$.

\section{Stronger Versions and Fractional Covers}

In this section we consider various conjectures that would imply Ryser's conjecture. We also consider versions involving the fractional covering number $\tau^{*}$. In a fractional cover, each vertex is assigned a non-negative real weight in such a way that the total weight on each edge is at least 1 . The fractional covering number $\tau^{*}$ is the least possible total of the vertex weights in a fractional cover.

The first author has thought for some time that the following stronger version of Ryser's conjecture might be true for intersecting hypergraphs:

Conjectute 3.1 In an intersecting $r$-partite hypergraph $H$ there exists a side of size $r-1$ or less, or a cover of the form $e \backslash\{x\}$, for some $e \in H$ and $x \in e$.

As we shall see shortly, a fractional version of Conjecture 3.1 is true. A natural stronger version of Conjecture 3.1 is that for each side $V_{i}$ either $\left|V_{i}\right|<r$ or there exists an edge $e$ such that $e \backslash V_{i}$ is a cover. However, this is false for $V_{1}$ in the following example. Let $H$ have a side $V_{1}$ of size $2^{r-2}$ and sides $V_{i}=\left\{a_{i}, b_{i}\right\}$ for $i>1$. The vertices $\left\{v_{P}\right\}$ of $V_{1}$ are indexed by the subsets $P \subseteq\{2, \ldots, r\}$ that contain the element 2. For each such $P$ there are two edges, $\left\{v_{P}\right\} \cup\left\{a_{i}: i \in P\right\} \cup\left\{b_{i}: i \notin P\right\}$ and $\left\{v_{P}\right\} \cup\left\{a_{i}: i \notin P\right\} \cup\left\{b_{i}: i \in P\right\}$. Conjecture 3.1 for general $r$-partite hypergraphs is:

Conjectute 3.2 In an r-partite hypergraph $H$ with $v(H)=k$ there exist sets $S_{1}, \ldots, S_{k}$, each of size at most $r-1$ and contained in a side or in an edge, such that $\bigcup_{i \leq k} S_{i}$ is a cover.

Another direction of strengthening Ryser's conjecture is a "biased" version. For a set $S$ of vertices write $|S|_{\text {bias }}$ for $\left|S \cap V_{r}\right|+\left|S \backslash V_{r}\right| /(r-1)$, where $V_{r}$ is the last side. In [19] the following was conjectured: in an $r$-partite hypergraph $H$ with sides $V_{1}, \ldots, V_{r}$ there exists a cover $C$ such that $|C|_{\text {bias }} \leq v(H)$. The motivation for this conjecture was that for $r=3$ this stronger version follows from the proof of the main result in [2]. A fractional version was proved in two different ways in two theses of students of the first author, [13] and [18]. Nevertheless, for $r>3$ the conjecture is false. The example showing it is a well known one; the family of cross-intersecting hypergraphs whose dual achieves the bound in the biclique edge colouring conjecture of Gyárfás and Lehel (see [7]). For $i=1, \ldots, r-2$ we take an edge $e_{i}$ that uses the first vertex on side $r$ and the $i$-th vertex on side $j$ for $j=1, \ldots, r-1$. Now for 
each permutation $\sigma$ of $\{1, \ldots, r-1\}$ add an edge that uses the second vertex on side $r$ and vertex $\sigma(j)$ on side $j$ for $j=1, \ldots, r-1$. Next, on each of the first $r-1$ sides break vertex $r-1$ apart so that all lines through it now go through a different vertex on that side. Neither of the two vertices on the last side are a cover on their own. Moreover, by [7], any cover that avoids using a vertex from the last side has size at least $2 r-4>r-1$. Hence the "biased" conjecture fails for all $r>3$.

However, a fractional version is true, which yields also fractional versions of Conjecture 3.2 and thus of Conjecture 3.1. An $r$-uniform hypergraph $H$ is said to be $(a, b)$-partitioned if $V(H)=V_{1} \cup V_{2}$, where $V_{1} \cap V_{2}=\emptyset$, and $\left|e \cap V_{1}\right|=a$ and $\left|e \cap V_{2}\right|=b$ for every $e \in H$.

Theorem 3.3 Given a $(1, r-1)$-partitioned hypergraph with sides $V_{1}, V_{2}$ there exist numbers $\beta_{u} \in\{0,1\}$ for each $u \in V_{1}$ and $\alpha_{e} \in \mathbb{R}^{+}$for each $e \in H$, such that:

1. $\sum_{u \in V_{1}} \beta_{u}+\sum_{e \in H} \alpha_{e} \leq v(H)$, and:

2. $\sum_{u \in V_{1}} \beta_{u} \chi_{\{u\}}+\sum_{e \in H} \alpha_{e} \chi_{e \backslash V_{1}}$ is a fractional cover for $H$.

Remark 3.4 The theorem implies that in a $(1, r-1)$-partitioned hypergraph $\tau^{*} \leq$ $(r-1) v$. This was already known, since Füredi [8] proved this inequality for any $r$-uniform hypergraph not containing a copy of the $r$-uniform projective plane.

For the proof we shall resort to topological notions, in particular that of "homological connectivity". A simplicial complex (or plainly a complex) is a closed downwards hypergraph, namely a collection of finite sets, called "simplices", containing with each simplex also all of its subsets. The homological connectivity $\eta_{H}(X)$ of a complex $X$ is the minimal $k$ for which all homology groups $H_{i}(X), i \leq k$, vanish, plus 2 (the addition of 2 simplifies the formulation of several results). Intuitively, $\eta_{H}(X)$ is the dimension of the smallest "hole" in $X$. In particular, $\eta_{H} \geq 1$ means ordinary connectivity of the complex. For example, if $X$ is a 1-dimensional complex (i.e., a graph) that is a cycle, then there is a hole of dimension 2, and no hole of dimension 1, and hence $\eta_{H}(X)=2$. We refer the reader to $[3,4,15]$ for some basic facts about connectivity.

Given a family $\mathcal{A}=\left(A_{1}, \ldots, A_{m}\right)$ of sets, a set formed by a partial choice function from the $A_{i}$ 's is said to be a (partial) rainbow set. A complete rainbow set is called a transversal. Given a complex $\mathcal{C}$ on $\bigcup_{i \leq m} A_{i}$ a rainbow set belonging to $\mathcal{C}$ is called an $(\mathcal{A}, \mathcal{C})$-transversal. The maximal size of a $(\mathcal{A}, \mathcal{C})$-transversal is denoted by $v(\mathcal{A}, \mathcal{C})$. We define the topological deficiency $\operatorname{def}(\mathcal{A}, \mathcal{C})$ as the maximum of $|I|-\eta_{H}\left(\mathcal{C}\left[\bigcup_{i \in I} A_{i}\right]\right)$ over all $I \subseteq[m]$. There is a topological deficiency version of Hall's theorem [6]:

Theorem $3.5 v(\mathcal{A}, \mathcal{C}) \geq m-\operatorname{def}(\mathcal{A}, \mathcal{C})$.

Theorem 3.6 If $\eta_{H}\left(\mathcal{C}\left[\bigcup_{i \in I} A_{i}\right]\right) \geq|I|-d$ for all $I \subseteq[m]$ then there exists a partial rainbow set belonging to $\mathcal{C}$ of size $m-d$.

The topological Hall theorem is the case $d=0$. It appears in [6] in a homotopical version (and even this, only implicitly), and explicitly in [15]. The case of general $d$ is obtained by the familiar device of adding "leeway". 
For any subset $S$ of $V$, we denote by $\chi_{S}$ the characteristic function of $S$. We shall need another definition, about a special type of fractional covers. Let

$$
\tau_{S}(H)=\min \left\{\sum_{e \in H} \alpha_{e}: \sum \alpha_{i} \chi_{e} \text { is a cover for } H\right\} .
$$

A result connecting these concepts is:

Theorem 3.7 [4] Let $H$ be a hypergraph and let $L(H)$ be its line graph. Then $\eta_{H}\left(\mathcal{I}(L(H)) \geq \tau_{s}(H)\right.$.

With the preliminaries at hand, we can now prove Theorem 3.3.

Proof Let $V_{1}=\left\{v_{1}, v_{2}, \ldots, v_{m}\right\}$. For $i \leq m$ let $A_{i}=\left\{f \subseteq V_{2} \mid\left\{v_{i}\right\} \cup f \in H\right\}$, and let $K=\bigcup_{i \leq m} A_{i}$. Let $\mathcal{C}=\mathcal{I}(L(K))$. So, the vertices of $\mathcal{C}$ are the $(r-1)$-tuples belonging to $K$ and the simplices are the matchings. Denote by $\Gamma$ the resulting ISR system. Clearly, $v(\Gamma)=v(H)$. Write $d$ for $\operatorname{def}(\Gamma)$. By Theorem 3.5, $v(\Gamma) \geq m-d$. Let $J$ be a subset of $[m]$ such that $d=|J|-\eta_{H}\left(\mathcal{C}\left[\bigcup_{i \in J} A_{i}\right]\right)$. By Theorem 3.7 we have $\tau_{s}\left(\bigcup_{i \in J} A_{i}\right) \leq|J|-d$, so there are numbers $\alpha_{e}, e \in K$ such that $\sum_{e \in K} \alpha_{e} \leq|J|-d$ and $\sum_{e \in K} \chi_{e}$ is a fractional cover for $\bigcup_{i \in J} A_{i}$. Taking $\beta_{u_{i}}=1$ for $i \notin J$ and $\beta_{u_{j}}=0$ for $j \in J$ completes the proof of the theorem.

In particular, if $v(H)=1$ then Theorem 3.3 says that either $\left|V_{1}\right|=1$ or there exists a fractional cover of size at most $r-1$, consisting of a linear combination with positive coefficients of characteristic functions of sets of the form $e \backslash V_{1}$.

Another stronger version of Ryser's conjecture, conjectured independently by Lovász [14] at around the same time as Ryser made his conjecture, is:

Conjectute 3.8 In an r-partite hypergraph $H$ there exists a set $S$ of vertices of size at most $r-1$, such that $v(H-S) \leq v(H)-1$.

The strengthening of Conjecture 3.8 along the lines of Conjecture 3.1 is:

Conjectute 3.9 In an $r$-partite hypergraph $H$ there exists a set $S$ of size $r-1$ or less, contained in an edge or in a side, whose removal reduces the matching number by at least 1 .

In the fractional case it is enough to take sets of the second type, those contained in an edge. To show this, we first prove a Lemma that is stronger than we actually need, but could be of independent interest.

Lemma 3.10 In every $r$-partite hypergraph $H$ there exists an optimal fractional cover in which at most one side has positive weight on all of its vertices.

Proof Associating each dimension of $\mathbb{R}^{n}$ with a vertex of $H$, let $Q$ be the polytope in $\mathbb{R}^{n}$ defined by $\mathbf{w} \chi_{e} \geq 1$ for all $e \in H$. Then $\tau^{*}(H)=\min \{\mathbf{w} \cdot \mathbf{1} \mid \mathbf{w} \in Q\}$ and the minimum is attained at a vertex $\mathbf{u}$ of $Q$. Suppose that there exist two distinct sides $V_{i}, V_{j}$ of $H$ such that $u(v)>0$ for every $v \in V_{i} \cup V_{j}$. We claim that $\left|V_{i}\right|=\left|V_{j}\right|$. To see this, assume to the contrary that (say) $\left|V_{i}\right|<\left|V_{j}\right|$. Now choose a positive 
$\epsilon \leq \min \left\{u(v) \mid v \in V_{j}\right\}$, and define $u^{\prime}(v)=u(v)-\epsilon$ for $v \in V_{j}, u^{\prime}(v)=u(v)+\epsilon$ for $v \in V_{i}$, and $u^{\prime}(v)=u(v)$ for $v \notin V_{i} \cup V_{j}$. Then $\mathbf{u}^{\prime}$ is a fractional cover of smaller size, contradicting the minimality property of $\mathbf{u}$. Having shown that $\left|V_{i}\right|=\left|V_{j}\right|$, we now take a number $\epsilon>0$ smaller than $\min \left\{u(v) \mid v \in V_{i} \cup V_{j}\right\}$, and note that $\mathbf{u}=\left(\mathbf{u}^{\prime}+\mathbf{u}^{\prime \prime}\right) / 2$, where $\mathbf{u}^{\prime}:=\mathbf{u}+\epsilon \chi_{V_{i}}-\epsilon \chi_{V_{j}}$ and $\mathbf{u}^{\prime \prime}:=\mathbf{u}-\epsilon \chi_{V_{i}}+\epsilon \chi_{V_{j}}$ are both fractional covers. This contradicts the fact that $\mathbf{u}$ is a vertex of $Q$.

We have shown that at least $r-1$ of the sides $V_{i}$ of $H$ contain a vertex $v$ of $H$ for which $u(v)=0$.

Theorem 3.11 In an $r$-partite hypergraph $H$ there exists a set $S=e \backslash\{v\}$ for some $v \in e \in H$, such that $v^{*}(H-S) \leq v^{*}(H)-1$.

Proof By Lemma 3.10 there exists an optimal fractional cover $u$ of $H$ and a vertex $v$ such that $u(v)=0$. Let $e$ be any edge of $H$ containing $v$, and let $S=e \backslash\{v\}$. Since $u$ is a fractional cover and $u(v)=0$, the weight of $u$ on $S$ is at least 1. Clearly, $u$ restricted to $V \backslash S$ is a fractional cover for $H-S$, proving the theorem.

Acknowledgments We thank Abu-Khazneh and Pokrovskiy [1] for sharing their paper and their ideas with us.

\section{References}

1. Abu-Khazneh, A., Pokrovskiy, A.: Intersecting Extremal Constructions in Rysers Conjecture for $r$ Partite Hypergraphs. http://arxiv.org/abs/1409.4938

2. Aharoni, R.: Ryser's conjecture for tripartite 3-graphs. Combinatorica 21, 1-4 (2001)

3. Aharoni, R., Berger, E.: The intersection of a matroid and a simplicial complex. Trans. Am. Math. Soc. 358, 4895-4917 (2006)

4. Aharoni, R., Berger, E., Meshulam, R.: Eigenvalues and homology of flag complexes and vector representations of graphs. Geom. Funct. Anal. 15, 555-566 (2005)

5. Aharoni, R., Charbit, P., Howard, D.: On a generalization of the Ryser-Brualdi-Stein conjecture. J. Graph Theory (to appear)

6. Aharoni, R., Haxell, P.: Hall's theorem for hypergraphs. J. Graph Theory 35, 83-88 (2000)

7. Chen, G., Fujita, S., Gyárfás, A., Lehel, J., Tóth, A.: Around a Biclique Cover Conjecture, arXiv.org:arXiv: 1212.6861

8. Füredi, Z.: Maximum degree and fractional matchings in uniform hypergraphs. Combinatorica 1, 155-162 (1981)

9. Haxell, P., Narins, L., Szabó, T.: Extremal Hypergraphs for Ryser's Conjecture I: Connectedness of Line Graphs of Bipartite Graphs, arXiv:1401.0169 [math.CO]

10. Haxell, P., Narins, L., Szabó, T.: Extremal Hypergraphs for Ryser's Conjecture II: Home-Base Hypergraphs. arXiv:1401.0171 [math.CO]

11. Haxell, P.E., Scott, A.D.: On Ryser's conjecture. Electron. J. Combin. 19(1), \#P23 (2012)

12. Kahn, J.: On a problem of Erdős and Lovász II: $n(r)=O(r)$. J. Am. Math. Soc. 7, 125-143 (1994)

13. Kfir, O.: Acyclic Systems of Representatives, M.Sc thesis, Technion (2006)

14. Lovász, L.: On Minimax Theorems of Combinatorics, Ph.D thesis, Matemathikai Lapok 26 (1975), 209-264 (in Hungarian)

15. Meshulam, R.: The clique complex and hypergraph matching. Combinatorica 21, 89-94 (2001)

16. Mansour, T., Song, C., Yuster, R.: A comment on Ryser's conjecture for intersecting hypergraphs. Graphs Combin. 25, 101-109 (2009)

17. Ryser, H.J.: Neuere Probleme der Kombinatorik. In: Vorträge über Kombinatorik, Oberwolfach, Matematisches Forschungsinstitute Oberwolfach, pp. 69-91, Germany (1967)

18. Zewi, N.: Vector Representation of Domination, M.Sc thesis, Technion (2010)

19. Zewi, N.: Vector representation of graph domination. J. Graph Theory 70, 152-170 (2012) 